\title{
How Choice Reveals and Shapes Expected Hedonic Outcome
}

\author{
Tali Sharot, ${ }^{1}$ Benedetto De Martino, ${ }^{1,2}$ and Raymond J. Dolan ${ }^{1}$ \\ ${ }^{1}$ Wellcome Trust Centre for Neuroimaging, Institute of Neurology, University College London, WCN 3BG London, United Kingdom, and ${ }^{2}$ Division of the \\ Humanities and Social Sciences, California Institute of Technology, Pasadena, California 91125
}

Humans tend to modify their attitudes to align with past action. For example, after choosing between similarly valued alternatives, people rate the selected option as better than they originally did, and the rejected option as worse. However, it is unknown whether these modifications in evaluation reflect an underlying change in the physiological representation of a stimulus' expected hedonic value and our emotional response to it. Here, we addressed this question by combining participants' estimations of the pleasure they will derive from future events, with brain imaging data recorded while they imagined those events, both before, and after, choosing between them. Participants rated the selected alternatives as better after the decision stage relative to before, whereas discarded alternatives were valued less. Our functional magnetic resonance imaging findings reveal that postchoice changes in preference are tracked in caudate nucleus activity. Specifically, the difference in blood oxygenation level-dependent (BOLD) signal associated with the selected and rejected stimuli was enhanced after a decision was taken, reflecting the choice that had just been made. This finding suggests that the physiological representation of a stimulus' expected hedonic value is altered by a commitment to it. Furthermore, before any revaluation induced by the decision process, our data show that BOLD signal in this same region reflects the choices we are likely to make at a later time.

\section{Introduction}

Actions not only reflect expectancies of hedonic outcome, but also alter such expectations (Ariely and Norton, 2008). A classic observation here is that after making a difficult choice between two equally valued options, such as between two desirable job offers, people subsequently value the selected alternative more strongly then they initially had, and the discarded one less so. This phenomena was first demonstrated experimentally in 1956 by psychologist Jack Brehm. Brehm reported that after picking between two similarly valued home appliances, housewives subsequently asserted that the appliance they selected was even better than they initially thought, and the rejected option was not that great after all (Brehm, 1956). Cognitive dissonance theory, one of the most influential theories in psychology, was introduced to account for this pattern of findings (Festinger, 1957). According to cognitive dissonance theory, having to make a choice between two similarly desirable alternatives engenders psychological discomfort. This is because the decision conflicts with the desirable aspects of the rejected alternative, and with the undesirable aspects of the selected alternative (Festinger, 1957). According to the theory such psychological tension is reduced by reevaluating the options postchoice (for an alternative account see Bem, 1967).

Brehm's experiment, known as the "free-choice paradigm", has been replicated numerous times (for a review see HarmonJones and Mills, 1999). Recently, this intriguing effect was also

Received 0ct. 15, 2008; revised Dec. 9, 2008; accepted Dec. 27, 2008.

This work was supported by a Wellcome Trust Programme Grant to R.J.D. and a British Academy Postdoctoral Fellowship to T.S. We thank J. McDermott for programming and discussion; G. Flandin for assistance with programming and analysis; K. Friston for advice on design and analysis; and P. Dayan, E. Phelps, S. Fleming, B. Strange, and P. Bossaerts for comments on an earlier version of this manuscript.

Correspondence should be addressed to Tali Sharot, Wellcome Trust Centre for Neuroimaging, Institute of Neurology, University College London, 12 Queen Square, London WCN 3BG, UK. E-mail: t.sharot@fil.ion.ucl.ac.uk.

D01:10.1523/JNEUROSCI.4972-08.2009

Copyright $\odot 2009$ Society for Neuroscience $\quad$ 0270-6474/09/293760-06\$15.00/0 shown in amnesic patients who did not even remember which option they had chosen (Lieberman et al., 2001), in young children (Egan et al., 2007), and in capuchin monkeys that had to choose between different colored chocolate M\&Ms (Egan et al., 2007). Although initially the monkeys valued all M\&Ms equally, like humans, they liked the unchosen M\&M less after a decision was made. These examples speak to the possibility that choice induced reevaluation is mediated by relatively automatic processes that do not necessarily depend on explicit memory (Lieberman et al., 2001) or highly evolved cognitive mechanisms (Egan et al., 2007).

Whether selecting M\&Ms, household appliances, jobs, or a potential spouse it is unknown whether the act of choosing actually modulates our physiological representation of a stimulus' expected hedonic value and our emotional response to it. If indeed this is the case, one would expect postchoice preference change to be observed in brain regions implicated in processing and predicting rewarding events, such as the striatum, nucleus accumbens, amygdala, and ventral medial and orbital prefrontal cortex (Delgado et al., 2003; Delgado, 2007; Seymour et al., 2007).

To investigate the relationship between choice and expected hedonic value, we adapted the free-choice paradigm (Brehm, 1956) to functional magnetic resonance imaging (fMRI). The study had two specific goals. First, we wanted to identify a neurobiological expression of postchoice changes in valuation. Second, we wished to address whether a choice between two equally rated alternatives is arbitrary or in fact predetermined by marginal differences in hedonic value representations that can be observed in the brain.

\section{Materials and Methods \\ Participants}

Fifteen volunteer participants were recruited through posted advertisements. One participant was eliminated from the analysis because of excessive number of trials with no response $(>25 \%)$. A second participant 
was eliminated because of usage of incorrect button keys leaving 13 participants (males $=8$, females $=5$; age range $=18-32$ ) in the analysis (for similar sample sizes see Phelps et al., 2004; Delgado et al., 2005; Daw et al., 2006; Kable and Glimcher, 2007; Hasson et al., 2008). Before scanning participants completed a screening form for significant medical conditions. All participants gave informed consent and were paid for their participation.

\section{Stimuli and procedure}

Overview. We obtained fMRI data while participants imagined taking a vacation in different destinations (e.g., Thailand, Greece). Participants were then asked to indicate how happy they estimated they would feel vacationing in each of these destinations. Subsequently, participants were presented with pairs of destinations they had rated equally, and were required to choose at which one they would rather take a vacation (2/3 of the trials, critical condition). In another condition participants were presented with easy choices involving presentation of locations they had rated unequally ( $1 / 3$ of the trials, noncritical condition). Participants were not aware during the first scanning session that they would be required to make these later choices. In a postchoice scanning session participants imagined and rated the destinations again.

Stimuli consisted of 80 names of vacation destinations. The order in which stimuli were presented was random. Before the scanning session participants went through four practice trials. The session began with a short structural scan, followed by four functional scan sessions. Finally, an additional longer structural scan was performed. Stimuli were presented via a mirror mounted on the head coil.

Scanning sessions. Scanning sessions 1 and 3 were $14 \mathrm{~min} 40 \mathrm{~s}$ each, and consisted of eighty trials of $11 \mathrm{~s}$. On each trial a name of a vacation destination appeared on screen for $6 \mathrm{~s}$. The participants were instructed to imagine themselves spending next year's vacation at that location. The participant then had $2 \mathrm{~s}$ to rate how happy they estimate they would be if they were to vacation at that location (1, unhappy; 2, a bit unhappy; 3 , neutral; 4 , happy; 5 , very happy; 6 , extremely happy). Responses were made using a button box in the left-hand for ratings $1-3$, and a button box in the right-hand for ratings $4-6$. If the participant did not respond that trial was excluded from the final data analysis. Finally a fixation cross was presented for $3 \mathrm{~s}$.

Reaction times (RTs) did not differ for subsequently chosen versus rejected stimuli in the critical condition in either session, and no interaction was found between decision (chosen/rejected) and scan (prechoice/postchoice). In the noncritical condition reaction times were shorter for subsequently chosen versus rejected stimuli in scan 3 $(p<0.05)$. Overall, reaction times were shorter in the third scanning session $(0.73 \mathrm{~s})$ than the first scanning session $(0.83 \mathrm{~s} ; p<0.05)$.

The order in which the stimuli was presented in the first scan was not related to subsequent choice (i.e., subjects were not more likely to chose stimuli that were imagined later in the scan or earlier in the scan) in the critical condition $(p>0.8)$, although a trend was observed in the noncritical condition $(p>0.07)$.

Scanning session 2 was 6 min long and consisted of forty trials of $9 \mathrm{~s}$ each. On each trial two names of vacation destinations from session 1 appeared on screen side by side for $4 \mathrm{~s}$. Then the word "choose" appeared on screen above the two options for 2 additional seconds. The participants were instructed to indicate which location they would prefer to vacation at next year by pressing one of two buttons on the right button box when the word "choose" came up. After making a response a star sign appeared next to the chosen location. Finally a fixation cross was presented for $3 \mathrm{~s}$. Choices were of course hypothetical, because participants did not actually receive the vacations they picked.

Pairs were determined by a Matlab program such that $\sim 2 / 3$ of the trials included two options that were rated the same in session 1 (the critical condition: difficult choice), and the rest ( $\sim 1 / 3$ of the trials) included two options that were rated differently in session 1 (the noncritical condition: easy choice). The number of trials in the critical condition was double than in the noncritical condition to maximize the power of finding differences pre- and postchoice in this condition of interest (difficult choice condition). Each stimulus appeared in only one pair. Reac- tion times for making the "difficult choices" $(0.6 \mathrm{~s})$ were longer than for making the "easy choices" $\left(0.5 \mathrm{~s} ; t_{(12)}=2.45 ; p<0.05\right)$.

Only behavioral data from this session was used in data analysis. Specifically, the choices made during this session were used to classify the trials in scan 1 and 3 into trials of subsequently (and previously) selected stimuli, and subsequently (and previously) rejected stimuli.

Scanning session 4 was a 4 min visual-motor task, distinct from the rest of our experiment, in which a fixation cross was presented for $6 \mathrm{~s}$ and than a circle was presented for $2 \mathrm{~s}$. The participants had to press the right button box each time the circle appeared. Data from this scan are not reported here.

Postscanning questions. After the scanning session, participants were asked to rate all stimuli on four scales: Previous visits (have you been to this location before? From $0=$ never to $6=$ lived there); Familiarity (Regardless of previous visits, how familiar is this location to you from books/TV/films? From $1=$ low to $6=$ high); Vividness (when you imagine going on vacation to this location how vivid is your image? From $1=$ low to $6=$ high); and Arousal (when you imagine going on vacation to this location how emotionally arousing is your image? From $1=$ low to 6 = high). Postscanning data of one participant were lost because of a computer error.

\section{Behavioral analysis}

First, the mean rating was calculated for each rating session and participant. Then the distance of each rating from the mean was computed for each trial, and the difference in scores between prechoice and postchoice ratings was calculated for each stimulus. These difference scores were then submitted to a 2 (decision: chosen/rejected) by 2 (condition: difficult choice/easy choice) ANOVA, followed by planned $t$ tests. The same analysis was implemented for the difference of raw scores between prechoice and postchoice ratings. Ratings from the four follow-up questions were also submitted to a 2 by 2 ANOVA as above (see supplemental Table 1 , available at www.jneurosci.org as supplemental material, for all scores).

\section{MRI scanning and data analysis}

The study was conducted at the Wellcome Trust Center for Neuroimaging at University College London using a 3T Siemens Allegra scanner equipped with a Siemens head coil. Anatomical images were acquired using MPRAGE scans, which were followed by 1-mm-thick axial slices parallel to the AC-PC plane. Functional scans used a gradient echo sequence, $\mathrm{TR}=2.7 \mathrm{~s}, \mathrm{TE}=30 \mathrm{~ms}, \mathrm{FA}=90$, matrix $=64 \times 64, \mathrm{FOV}=192$ $\mathrm{mm}$, slice thickness $=2 \mathrm{~mm}$. A total of 42 axial slices $\left(-30^{\circ}\right.$ tilt $)$ were sampled for whole brain coverage. The in-plane resolution was $3 \mathrm{~mm} \times$ $3 \mathrm{~mm}$.

Imaging data were analyzed for the rating sessions (scans 1 and 3). Statistical Parametric Mapping (SPM5; Wellcome Trust Centre for Neuroimaging, London, UK, http://www.fil.ion.ucl.ac.uk/spm) was used to analyze the fMRI data. Images were realigned with the first volume (after discarding the first six dummy volumes) and unwarped, normalized to a standard EPI template based on the Montreal Neurological Institute (MNI) reference brain, resampled to $2 \times 2 \times 2 \mathrm{~mm}^{3}$ voxels, and spatially smoothed (8 mm FWHM).

For all analyses we report results in a priori regions of interest (striatum, nucleus accumbens, amygdala, OFC, VMPFC, anterior cingulate cortex) previously identified in neuroimaging studies of reward processing, reward expectation (O'Doherty et al., 2002; Delgado et al., 2003; Gottfried et al., 2003; O'Doherty, 2004; Delgado, 2007; Seymour et al., 2007), and simulation of future positive events (Sharot et al., 2007; D'Argembeau et al., 2008). Activations in other regions are reported in the supplemental tables, available at www.jneurosci.org as supplemental material.

Two main analyses were performed. First, for each participant a time series was created indicating the temporal position of stimuli onset (the appearance of the vacation destination name) to stimuli offset. These time series were included in a parametric modulation analysis (random effects GLM) on all trials from session 1 and 3 using the participants' ratings on each trial as the covariate in an ANCOVA $(p<0.0005$, uncorrected; $>150$ contiguous voxels). A relatively stringent threshold was 
used, because the power for this analysis was greater than all subsequent analyses, since all 160 trials were included. Trials were modeled as $6 \mathrm{~s}$ "mini blocks".

Second, trials from session 1 (prechoice) and session 3 (postchoice) were classified into six groups according to the participants' choices during session 2. These included the four critical groups from the difficult choice condition: (1) selected stimuli prechoice (trials in session 1 of stimuli that were later presented in the difficult choice condition in session 2 and selected by the participant out of the two options), (2) rejected stimuli prechoice (trials in session 1 of stimuli that were later presented in the difficult choice condition in session 2 and rejected by the participant), (3) selected stimuli postchoice (trials in session 3 of stimuli that were previously presented in the difficult choice condition in session 2 and selected), (4) rejected stimuli postchoice (trials in session 3 of stimuli that were previously presented in the difficult choice condition in session 2 and rejected). For the non critical condition (easy choice), data from sessions 1 and 3 were combined. Because the number of trials in the noncritical condition was one-half than in the critical condition (difficult choice) collapsing allowed an equal amount of trials across the different bins, and a sufficient number of trials for data analysis in the noncritical condition. Thus, the two remaining bins were: selected stimuli noncritical condition (trials in session 1 and 3 of stimuli that were presented in the easy choice condition in session 2 and selected), rejected stimuli noncritical condition (trials in session 1 and 3 of stimuli that were presented in the easy choice condition in session 2 and rejected). For each participant a time series was created indicating the temporal position of the six different trial types from stimuli onset to stimuli offset.

Random effects GLM analyses were conducted on the data of all participants ( $p<0.001$; uncorrected). First, we contrasted blood oxygenation level-dependent (BOLD) signal during each rating scan (prechoice and postchoice) of trials of stimuli that were selected in the difficult choice, with trials of stimuli that were rejected. Second, we conducted an interaction to identify regions in which the difference in BOLD signal between selected and rejected stimuli was greater after the choice was made relative to before. A $4 \mathrm{~mm}^{3}$ cube was defined centered around the peak of activation identified in the above interaction. Within this region we conducted an analysis of covariance contrasting postchoice BOLD signal differences between selected and rejected stimuli with prechoice differences, using each participant's spread in postchoice rating as a covariate $[p<0.05$, familywise error (FWE) small volume corrected].

For anatomical region of interest (ROI) analysis we extracted the mean parameter estimates averaged across the whole anatomically defined ROIs for the different trial types, and performed statistical tests on these values. These regions were defined according to the Talairach Daemon atlas (Lancaster et al., 1997) using the SPM WFU PickAtlas tool (Maldjian et al., 2003).

To identify other voxels in the brain that showed greater BOLD responses during one trial type than the other, we conducted a whole-brain exploratory analysis on group data $(p<0.001$, uncorrected) using a random effects GLM.

\section{Results}

Consistent with Brehm's classic results (Brehm, 1956), all our participants changed their valuations of the options after choosing between alternatives they had initially rated the same. Specifically, mean-corrected hedonic ratings increased after the decision stage for selected stimuli $\left(t_{(12)}=7.67 ; p<0.0001\right)$ and decreased for rejected stimuli $\left(t_{(12)}=2.8 ; p<0.02\right)$. Although the mean expected hedonic outcome of selected and rejected stimuli in the difficult choice condition did not differ before a choice was made, significant differences emerged postchoice $\left(t_{(12)}=18.1\right.$; $p<0.0001)$. In contrast, ratings of stimuli presented in the easy choice condition, in which a choice was made between two differently rated stimuli, remained unchanged postchoice. Figure 1 portrays the results of the analysis described above for the difference scores between prechoice and postchoice mean-corrected ratings. The same analysis was implemented for the difference of

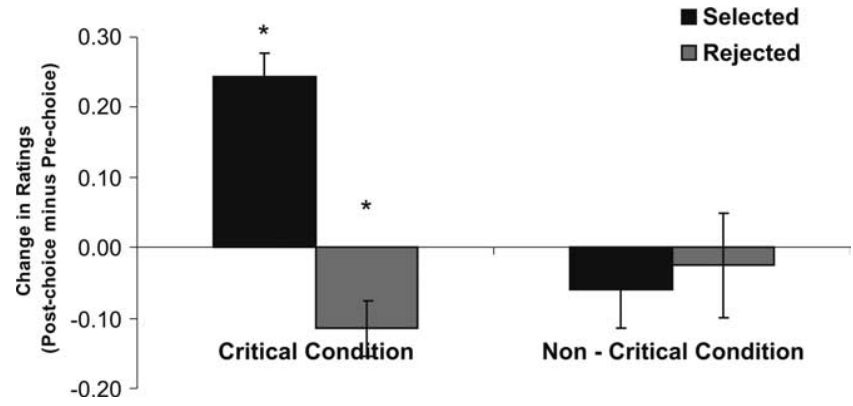

Figure 1. Postchoice changes in estimated hedonic experience. When a difficult choice was made between two equally rated stimuli (critical condition) ratings of estimated happiness increased postchoice for selected stimuli, and decreased for rejected stimuli. The bars represent the difference in mean corrected ratings from prechoice scan to postchoice scan. These are significantly different from zero only in the critical condition. When an easy choice was made between two differently rated stimuli (noncritical condition) ratings for both selected and rejected stimuli remained the same before and after choice. Error bars represent SEM. *Significantly different from zero ( $p<0.05$, two tailed).

raw scores between prechoice and postchoice ratings, revealing the same pattern of results. The mean scores for all ratings (including postscanning ratings) and statistical analysis are presented in supplemental Table 1, available at www.jneurosci.org as supplemental material.

We next examined whether BOLD responses in regions implicated in reward processing tracked expectations of future hedonic reaction during imagination. Using hedonic ratings as a covariate, we conducted a parametric modulation analysis on data from all 160 prechoice and postchoice trials $(p<0.0005$, uncorrected; $>150$ contiguous voxels). Significant positive correlations between BOLD signal and hedonic ratings were found in the caudate nucleus bilaterally (Talairach coordinates of peak voxel: R: 16, 24, 8 and 22, -16, 25; L: $-10,22,1$ ) (Fig. 2a), a region previously related to reward processing and reward anticipation (Delgado, 2007). We also observed significant effects in left amygdala $(-22,-8,-8)$ (Fig. $2 b$ ) as well as in left pregenual anterior cingulate cortex $(-14,48,-6)$ (Fig. $2 c$ ). All of these regions have previously been shown to be engaged when participants imagined positive future events (Sharot et al., 2007; D'Argembeau et al., 2008). For exploratory parametric modulation analysis outside the ROIs, see supplemental Table 2, available at www.jneurosci.org as supplemental material.

To test whether activity in these regions specifically tracked differences in estimated hedonic reaction to the stimuli, rather than other potential differences inherent to the stimuli, we included ratings of arousal, vividness, familiarity, and previous visits to these locations as covariates in our parametric modulation analysis. This more stringent analysis revealed that activity in the caudate nucleus bilaterally correlated with estimated hedonic reaction even when controlling for ratings of arousal, vividness, familiarity, and previous visits ( $p<0.0005,>150$ voxels) (Fig. $3 a)$. In contrast, these additional regressors accounted for sufficient variance in the response of amygdala and pregenual anterior cingulate voxels that the correlation with expected hedonic reaction was no longer significant at this threshold. Thus, during imagination of a future event, BOLD signal in the caudate nucleus appears to specifically track a physiological signature of estimated hedonic reaction.

Next, we asked whether activity in areas related to reward processing reflect subsequent choice. To test this, we contrasted the BOLD signal from the prechoice scan for trials of stimuli that were later selected with that for trials of stimuli that were later 
a.
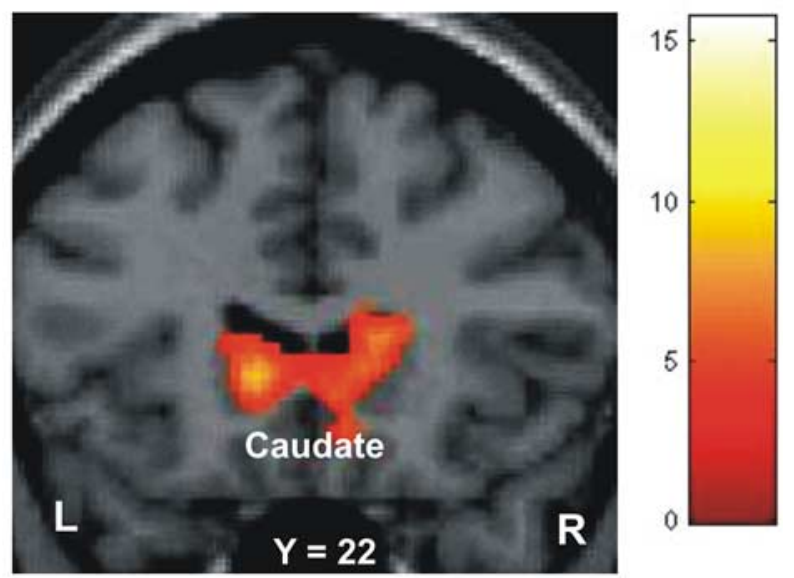

b.

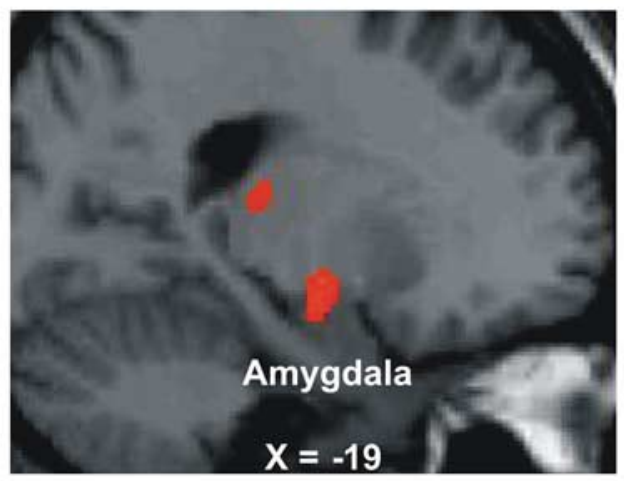

c.

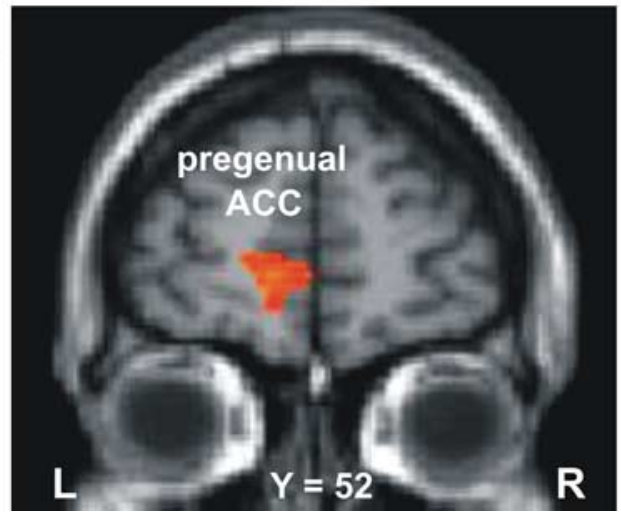

Figure 2. $\boldsymbol{a}-\boldsymbol{c}$, Brain activity related to estimated hedonic experience. Regions in which BOLD response was positively correlated with participants' ratings of estimated hedonic experience ( $p<0.0005$, uncorrected; $>150$ contiguous voxels) included the right and left caudate nucleus, previously associated with reward expectancy (Knutson et al., 2001; Gottfried et al., 2003) (a), as well as the left amygdala ( $\boldsymbol{b}$ ), and left pregenual anterior cingulate cortex (c), both previously shown to be engaged when imagining positive future autobiographical events (Sharot et al., 2007; D’Argembeau et al., 2008).

rejected in the difficult choice condition. Note that this comparison is between stimuli that had been given the same ratings of estimated hedonic reaction prechoice. We found greater BOLD signal in the right and left caudate nucleus for stimuli that were subsequently selected in the difficult choice versus those subsequently rejected (peak voxel: R: $6,2,12$; L: $-6,4,12 ; p<0.001$, uncorrected). The mean parameter estimate of BOLD signal averaged over the whole anatomically defined right caudate nucleus was significantly greater for stimuli later selected in the difficult choice relative to stimuli that were later rejected $(p<0.05)$ (Fig. $3 b)$. No significant effects were found in any other a priori regions of interest for this contrast, nor did any voxels in these regions show the opposite effect (i.e., where activity was greater for stimuli later rejected vs stimuli later selected). The clear implication is that during the initial imagination session, differences in BOLD response within the caudate nucleus reflect subsequent choice.

Our behavioral results demonstrated that after making choices the participants' assessments of the alternatives changed to fit more closely with their decisions. Specifically, ratings of estimated hedonic reaction were greater for selected stimuli relative to rejected stimuli only after a choice was made (i.e., during the last session). To examine whether these changes were also reflected in BOLD signal, we tested for an interaction that highlights areas showing a greater difference in activity between selected and rejected stimuli after a difficult choice was made (i.e., in the last scan) relative to before (i.e., in the first scan). This critical analysis revealed a significant effect in right caudate nucleus (peak voxel: $20,-24,24 ; p<0.001$, uncorrected) (see supplemental note, available at www.jneurosci.org as supplemental material). To ensure this interaction was not restricted to dorsal posterior region of the caudate, which is somewhat distant from the caudate regions that typically show reward effects in other studies (Delgado, 2007), and is distant from the peak voxel showing a correlation with hedonic ratings in the present study, we used the results of the parametric modulation analysis to define a small volume in a more anterior/ventral region of the caudate nucleus. Specifically, we defined a cluster $(p<0.001$, uncorrected) in anatomically defined right caudate nucleus that showed the greatest correlation with hedonic ratings after controlling for ratings of arousal, vividness, familiarity, and previous visits. Examining within this cluster revealed a significant interaction (peak voxel: 10, 22, $0 ; p<0.05$, FWE small volume corrected).

Furthermore, BOLD signal in the right caudate nucleus during the last scan was greater while imagining previously chosen stimuli, relative to previously rejected stimuli in the critical condition (peak voxel: $14,-4,22 ; p<0.001$, uncorrected), and the mean parameter estimate of BOLD signal averaged over the whole anatomically defined right caudate nucleus was greater for stimuli that were previously selected in the difficult choice relative to stimuli that were previously rejected $(p<0.05)$ (Fig. $3 d$ ). Across individuals, the difference in BOLD signal between selected and rejected stimuli during the postchoice scan relative to the prechoice scan correlated with participants' postchoice change in ratings (peak voxel: $18,-20,26 ; r=0.63 ; p<0.05$, FWE small volume correction using a $4 \mathrm{~mm}^{3}$ cube centered around the peak interaction contrast in the caudate) (Fig. $3 d$ ). Thus, participants who were more inclined to reevaluate their ratings postchoice showed greater changes in caudate nucleus activity.

No significant interaction was found in any other a priori region, nor did any of these regions show the opposite effect (i.e., where difference in activity between selected and rejected stimuli were greater prechoice than postchoice). Together, these findings suggest that activity in the caudate nucleus tracks expected hedonic reactions during imagination, reflects subsequent choice, and importantly, expresses postchoice changes in expected hedonic value. For exploratory analysis outside the ROIs in the critical condition, see supplemental Table 3, available at www.jneurosci.org as supplemental material, and for results in the noncritical condition see supplemental Table 4, available at www.jneurosci.org as supplemental material. 


\section{Discussion}

After making a decision, humans often change their existing preferences to align more closely with the choice they had just made. Our behavioral results demonstrate that even when making hypothetical choices, with no apparent consequence, those decisions change assessments of the hedonic outcome of the alternatives. Specifically, after making a theoretical choice between two equally rated vacation destinations, participants deflated the valuation of the unchosen options while inflating evaluations of the chosen options.

Using fMRI we have identified the physiological expression of these choiceinduced preference modifications. Our findings reveal that postchoice changes in preference are reflected in caudate nucleus activity, suggesting that the biological representation of a stimulus' expected hedonic value is altered by a commitment to it. Furthermore, before any revaluation induced by the decision process, our data show that BOLD signal in this same region reflects the choices we are likely to make.

The caudate nucleus is one of the major targets for the brain's dopaminergic system (Wise and Rompre, 1989), and has a well documented role in reward processing, reward expectation and reward learning (Delgado, 2007). For example, increased activity in the caudate is associated with anticipation of both primary (Gottfried et al., 2003) and monetary (Knutson et al., 2001) rewards. In addition, two recent studies have reported increased activation in the caudate nucleus when imagining positive future events relative to negative future events (Sharot et al., 2007; D’Argembeau et al., 2008).

Consistent with these previous studies, in the current experiment the caudate nucleus emerged as the key brain region that tracked participants' ratings of expected hedonic consequences. Thus, the finding that activity in the caudate nucleus is greater while participants imagined vacationing in destinations they later selected relative to those they later rejected, before even knowing they will be required to make choices, suggests that the decision between equally rated destinations was not arbitrary. Rather, the decision was seemingly predetermined by differences in expected hedonic outcome as reflected in caudate activity during imagination. The caudate nucleus seems to be coding hedonic preference with a greater sensitivity than the rating scale used in the study. Note, that our data cannot determine whether the caudate nucleus response is reflective of hedonic experience during imagination (i.e., the pleasure we feel at the moment from imagining ourselves on a beach in Thailand), or strictly representing expectancies of hedonic outcome that are not experienced in the actual present.

The differences in BOLD signal associated with subsequent choice were not captured in the initial preference rating. We note that this finding grounds in neurobiology an explicit prediction from revealed preference theory in microeconomics (Samuelson, 1938), where it is axiomatic that preferences are most reliably indexed by the observable actions of a consumer (i.e., purchasing choices). Our findings do not necessarily imply that differences in preference are not consciously accessible prechoice. Indeed, the very fact that a preference was not expressed behaviorally in the prechoice scan does not mean that it was not experienced. For example, these differences may not have been large enough prechoice to be captured behaviorally using standard ratings. Importantly, however, postchoice differences in preferences became large enough to be observed using the same rating scale. The critical finding is that after a decision was made, the difference in caudate nucleus activity associated with the selected, versus the rejected, option was further enhanced. This difference was now revealed in participants' explicit behavioral assessment of hedonic outcome. The finding that postchoice change in ratings is reflected in a parallel change in the activity profile within the caudate nucleus suggests that the stimulus' representation of expected hedonic value is altered by choice.

Imagination serves an important adaptive function by allowing us to simulate and predict the outcome of an infinite number of possible future scenarios. Such flexibility goes beyond what can be learned from behavior alone, and allows us to prepare for what is yet to come. Specifically, simulating future events enables us to "prefeel" the pleasures and pains those events are likely to engender. These responses can be used as proxies for the actual hedonic reactions we are likely to experience should those events actually occur, and thus guide which actions to avoid and which to execute (Gilbert and Wilson, 2007). This ability is especially crucial for decision making in instances when the value of possible options are not easily distinguishable.

In accord with the above model, the current study highlights how the brain predicts an event's hedonic consequence, suggesting that during imagination a response generated in a subcortical 
region strongly implicated in reward processing conveys expected pleasure from the simulated event. This signal reflects a later action when an individual is faced with a difficult decision between two apparently equally desirable options. What is intriguing is that this signal is altered by the ensuing choice such that the difference in caudate nucleus activity associated with the selected and discarded stimuli is enhanced after a decision is taken, reflecting the choice that had just been made.

Although it is possible that postchoice reevaluation is driven by the need to reduce cognitive dissonance (Festinger, 1957), it is also feasible that reevaluation occurs because envisioning competing possibilities provides a new context in which the stimuli are assessed. Specifically, making a choice highlights the unique aspects of the two alternatives (Tversky, 1972; Houston et al., 1991). Thus, the act of choosing may change preference by providing new weights to aspects of the stimuli that may not have been considered thoroughly before.

Our findings suggest a model in which explicit postchoice reevaluation involves modification of the expected affective hedonic response to the stimulus. This mechanism may serve an adaptive purpose by increasing an individual's commitment to the action taken. It is interesting to speculate that in the absence of a rapid update of value that concurs with choice, we are likely to second-guess our decisions and actions, which may promote negative affect and interfere with our daily function.

\section{References}

Ariely D, Norton MI (2008) How actions create—not just reveal—preferences. Trends Cogn Sci 12:13-16.

Bem DJ (1967) Self-perception: an alternative interpretation of cognitive dissonance phenomena. Psychol Rev 74:183-200.

Brehm JW (1956) Post-decision changes in the desirability of choice alternatives. J Abnorm Soc Psychol 52:384-389.

D’Argembeau A, Xue G, Lu ZL, Van der Linden M, Bechara A (2008) Neural correlates of envisioning emotional events in the near and far future. Neuroimage 40:398-407.

Daw ND, O’Doherty JP, Dayan P, Seymour B, Dolan RJ (2006) Cortical substrates for exploratory decisions in humans. Nature 7095:876-879.

Delgado MR (2007) Reward-related responses in the human striatum. Ann N Y Acad Sci 1104:70-88.

Delgado MR, Locke HM, Stenger VA, Fiez JA (2003) Dorsal striatum responses to reward and punishment: effects of valence and magnitude manipulations. Cogn Affect Behav Neurosci 3:27-38.

Delgado MR, Frank RH, Phelps EA (2005) Perceptions of moral character modulate the neural systems of reward during the trust game. Nat Neurosci 8:1611-1618.
Egan LC, Santos LR, Bloom P (2007) The origins of cognitive dissonance: evidence from children and monkeys. Psychol Sci 18:978-983.

Festinger L (1957) A theory of cognitive dissonance. Stanford: Stanford UP.

Gilbert DT, Wilson TD (2007) Prospection: experiencing the future. Science 317:1351-1354.

Gottfried JA, O'Doherty J, Dolan RJ (2003) Encoding predictive reward value in human amygdala and orbitofrontal cortex. Science 301:1104-1107.

Harmon-Jones E, Mills J (1999) Cognitive dissonance progress on a pivotal theory in social psychology. Washington, DC: Braun-Brumfield.

Hasson U, Furman O, Clark D, Dudai Y, Davachi L (2008) Enhanced intersubject correlations during movie viewing correlate with successful episodic encoding. Neuron 57:452-462.

Houston DA, Sherman SJ, Baker SM (1991) Feature matching, unique features, and the dynamics of the choice process: predecision conflict and postdecision satisfaction. J Exp Soc Psychol 27:411-414.

Kable JW, Glimcher PW (2007) The neural correlates of subjective value during intertemporal choice. Nat Neurosci 10:1625-1633.

Knutson B, Adams CM, Fong GW, Hommer D (2001) Anticipation of increasing monetary reward selectively recruits nucleus accumbens. J Neurosci 21:RC159:1-5.

Lancaster JL, Rainey LH, Summerlin JL, Freitas CS, Fox PT, Evans AC, Toga AW, Mazziotta JC (1997) Automated labeling of the human brain: a preliminary report on the development and evaluation of a forwardtransform method. Hum Brain Mapp 5:238-242.

Lieberman MD, Ochsner KN, Gilbert DT, Schacter DL (2001) Do amnesics exhibit cognitive dissonance reduction? The role of explicit memory and attention in attitude change. Psychol Sci 12:135-140.

Maldjian JA, Laurienti PJ, Kraft RA, Burdette JH (2003) An automated method for neuroanatomic and cytoarchitectonic atlas-based interrogation of fMRI data sets. Neuroimage 19:1233-1239.

O'Doherty JP (2004) Reward representations and reward-related learning in the human brain: insights from neuroimaging. Curr Opin Neurobiol 14:769-776.

O’Doherty JP, Deichmann R, Critchley HD, Dolan RJ (2002) Neural responses during anticipation of a primary taste reward. Neuron 33:815-826.

Phelps EA, Delgado MR, Nearing KI, LeDoux JE (2004) Extinction learning in humans: role of the amygdala and vmPFC. Neuron 16:897-905.

Samuelson P (1938) A note on the pure theory of consumers' behavior. Economica 5:61-71.

Seymour B, Daw N, Dayan P, Singer T, Dolan RJ (2007) Differential encoding of losses and gains in the human striatum. J Neurosci 27:4826-4831.

Sharot T, Riccardi AM, Raio CM, Phelps EA (2007) Neural mechanisms mediating optimism bias. Nature 450:102-105.

Tversky A (1972) Elimination by aspects: a theory of choice. Psychol Rev 79:281-299.

Wise RA, Rompre PP (1989) Brain dopamine and reward. Annu Rev Psychol 40:191-225. 